\title{
Effect of Infection Timing on Fusarium Head Blight and Mycotoxin Accumulation in Open- and Closed-Flowering Barley
}

\author{
Megumi Yoshida, Naoyuki Kawada, and Takashi Nakajima
}

Research Team for Fusarium Head Blight Control, National Agricultural Research Center for Kyushu Okinawa Region (KONARC), 2421 Suya, Koshi, Kumamoto 861-1192, Japan.

Accepted for publication 5 April 2007.

\begin{abstract}
Yoshida, M., Kawada, N., and Nakajima, T. 2007. Effect of infection timing on Fusarium head blight and mycotoxin accumulation in openand closed-flowering barley. Phytopathology 97:1054-1062.

Barley has two flowering types, chasmogamous (open-flowering) and cleistogamous (closed-flowering). We examined the effect of the timing of Fusarium graminearum infection on Fusarium head blight (FHB) and mycotoxin accumulation in barley cultivars with different flowering types using greenhouse experiments. In the first experiment, 13 cultivars were spray inoculated at two different developmental stages, and the severity of FHB was evaluated. The effect of the timing of infection differed among cultivars. Cleistogamous cultivars were resistant at anthesis but susceptible at 10 days after anthesis, whereas chasmogamous cultivars were already susceptible at anthesis. In the second experiment, five cultivars
\end{abstract}

ABSTRACT were inoculated at three different developmental stages and the concentrations of deoxynivalenol (DON) and nivalenol (NIV) in mature grain were analyzed. Cleistogamous cultivars accumulated more mycotoxins (DON and NIV) when inoculated 10 or 20 days after anthesis than when inoculated at anthesis, whereas chasmogamous cultivars accumulated more mycotoxins when inoculated at anthesis. Thus, the most critical time for $F$. graminearum infection and mycotoxin accumulation in barley differs with cultivar, and likely is associated with the flowering type. Late infection, even without accompanied FHB symptoms, was also significant in terms of the risk of mycotoxin contamination.

Additional keywords: anther, cleistogamy, Gibberella zeae, Hordeum vulgare, scab, trichothecene.
Fusarium head blight (FHB) or scab, which is caused by several Fusarium spp., primarily Fusarium graminearum Schwabe (teleomorph Gibberella zeae (Schwein.) Petch), is a widespread and destructive disease of barley (Hordeum vulgare L.) and wheat (Triticum aestivum L.) $(18,22,32)$. This disease infects spikes and reduces grain yield and quality. Moreover, Fusarium spp. that cause FHB produce mycotoxins such as deoxynivalenol (DON), nivalenol (NIV), and zearalenone, which are toxic to humans and animals. Contamination of barley and wheat by these mycotoxins has been reported worldwide $(25,37,38)$.

$F$. graminearum can be divided into two chemotaxonomic groups: the DON chemotype and NIV chemotype $(9,13,30,34)$. DON is the most prevalent mycotoxin in cereals $(4,25)$ and DON chemotypes of Fusarium spp. prevail worldwide, whereas NIV chemotypes are more restricted $(12,21)$; therefore, greater attention generally is focused on DON than NIV as a trichothecene mycotoxin. A provisional maximum tolerable daily intake (PMTDI) of $1 \mu \mathrm{g}$ of DON per kilogram of body weight was established by the Joint FAO/World Health Organization Expert Committee on Food Additives in 2001 (4). However, NIV is also an important Fusarium mycotoxin that frequently occurs worldwide $(25,37)$ and commonly co-occurs with DON $(25,37,38$, $43,44)$. Moreover, NIV is more toxic to animals than DON $(28,40)$. Accordingly, control measures that are effective for both NIV and DON should be established.

To control FHB and mycotoxin contamination effectively, the critical timing of infection must be known. Wheat is most sus-

Corresponding author: T. Nakajima; E-mail address: ntakashi@affrc.go.jp

doi:10.1094/PHYTO-97-9-1054

This article is in the public domain and not copyrightable. It may be freely reprinted with customary crediting of the source. The American Phytopathological Society, 2007. ceptible to FHB during anthesis $(1,22,26,35)$, which likely is due to anther extrusion at that stage, because the initial Fusarium infection commonly occurs on extruded anthers (26). Whereas wheat is generally chasmogamous (open-flowering type), barley has both chasmogamous and cleistogamous (closed-flowering type) cultivars; in the latter, the florets do not open and anthers are not extruded at anthesis.

In Japanese barley cultivars, there is a strong association between row type and flowering type; most two-rowed cultivars are cleistogamous, whereas most six-rowed cultivars are chasmogamous. In most cases, the former are relatively resistant and the latter are susceptible to FHB (42), which is consistent with the general observation that two-rowed types are more resistant than six-rowed types $(3,7,17,32,36,47)$. Cleistogamy, rather than the row type, contributes to the resistance of barley cultivars to FHB, at least for infection that occurs at anthesis (42).

In our recent preliminary tests, however, high levels of DON and NIV contamination occurred, even in resistant two-rowed cleistogamous cultivars. In cleistogamous cultivars, anthers do not protrude at anthesis; however, they may be pushed out from the tips of florets by the developing grain several days after anthesis (2). In Japan, this phenomenon is observed commonly in cleistogamous cultivars. Thus, the timing of anther extrusion differs with flowering type. Therefore, the stage most susceptible to Fusarium infection may differ among barley cultivars.

McCallum and Tekauz (16) previously reported differential susceptibility to FHB at different developmental stages in four barley cultivars using spray inoculations. However, the effects of cultivar, timing of inoculation, and their interaction were significant for the colonization of seed by $F$. graminearum but not for visual symptoms of infection (16). The effect of the timing of $F$. graminearum infection on FHB in barley has not been well studied otherwise; to our knowledge, the effect of the timing of infection on mycotoxin contamination in barley has not been investigated. 
The objective of this study was to investigate the effect of the timing of infection by $F$. graminearum on FHB severity and the accumulation of DON and NIV in barley cultivars with different flowering types. Such knowledge will be helpful in developing effective control measures to reduce the risk of FHB and mycotoxin contamination in barley.

\section{MATERIALS AND METHODS}

Evaluation of FHB resistance at two developmental stages in barley cultivars (experiment 1). Thirteen barley cultivars differing in row type and flowering type (Fig. 1) were evaluated for FHB resistance at two developmental stages: anthesis and 10 days after anthesis (DAA). The experiment was conducted in Chikugo $\left(33.1^{\circ} \mathrm{N}, 130.3^{\circ} \mathrm{E}\right)$, Fukuoka, Japan, in 2004 and 2005, although only 8 of the 13 cultivars were tested in 2004. The sixrowed cultivars were chasmogamous and the two-rowed cultivars were cleistogamous, except for the two-rowed chasmogamous 'Satsuki Nijo.' 'Chevron,' the only non-Japanese cultivar tested, is relatively resistant to FHB among six-rowed barley cultivars $(3,17,27,32)$. The two-rowed cvs. Asahi 5, Seijo 17, and Nitta Nijo16 also are highly resistant (36). The other eight cultivars were representative samples of the current barley cultivars available for production in Japan. 'Sanuki hadaka' is a hull-less type, whereas the other 12 cultivars are all of the hulled type.
The cultivars were seeded in 15-cm-diameter plastic pots (four plants per pot) at the end of November 2003 and 2004, and were grown in a greenhouse under natural light until inoculation (March to April 2004 and 2005). The potting soil was prepared by mixing 25 liters of volcanic ash soil, 15 liters of sand, 10 liters of vermiculite, 10 liters of compost, and $75 \mathrm{ml}$ of calcium magnesium carbonate. Each pot contained $\approx 1$ liter of the mixed soil and was fertilized at the seedling growth stage (Zadoks growth stage [ZGS] 13) (46) and the stem elongation stage (ZGS 30 to 31 ) with 2.5 and $1.5 \mathrm{~g}$, respectively, of $\mathrm{N}-\mathrm{P}_{2} \mathrm{O}_{5}-\mathrm{K}_{2} \mathrm{O}$ at 14-14-14.

$F$. graminearum isolate $\mathrm{H}-3$ was used as the inoculum. Macroconidia of the isolate were produced by growing the fungus in mung bean liquid medium prepared as follows: $20 \mathrm{~g}$ of mung bean were boiled in 1 liters of water for $20 \mathrm{~min}$, and $1 \mathrm{~g}$ of yeast extract was added; the broth then was strained through a colander and autoclaved. The macroconidia suspension obtained was filtered to remove hyphae and was centrifuged at 7,000 rpm for $10 \mathrm{~min}$. After removal of the liquid medium, the pellet was suspended in distilled water and the macroconidia concentration was provisionally adjusted to $5 \times 10^{7}$ spores $/ \mathrm{ml}$ and then stored at $-80^{\circ} \mathrm{C}$ until use. On the days of inoculation, the macroconidia suspension was thawed at $22 \pm 2{ }^{\circ} \mathrm{C}$ (room temperature) and diluted to $5 \times 10^{5}$ spores $/ \mathrm{ml}$ with distilled water.

Inoculation and FHB evaluation were performed using the "pot-plant" method $(41,42)$, with some modification. Four (in

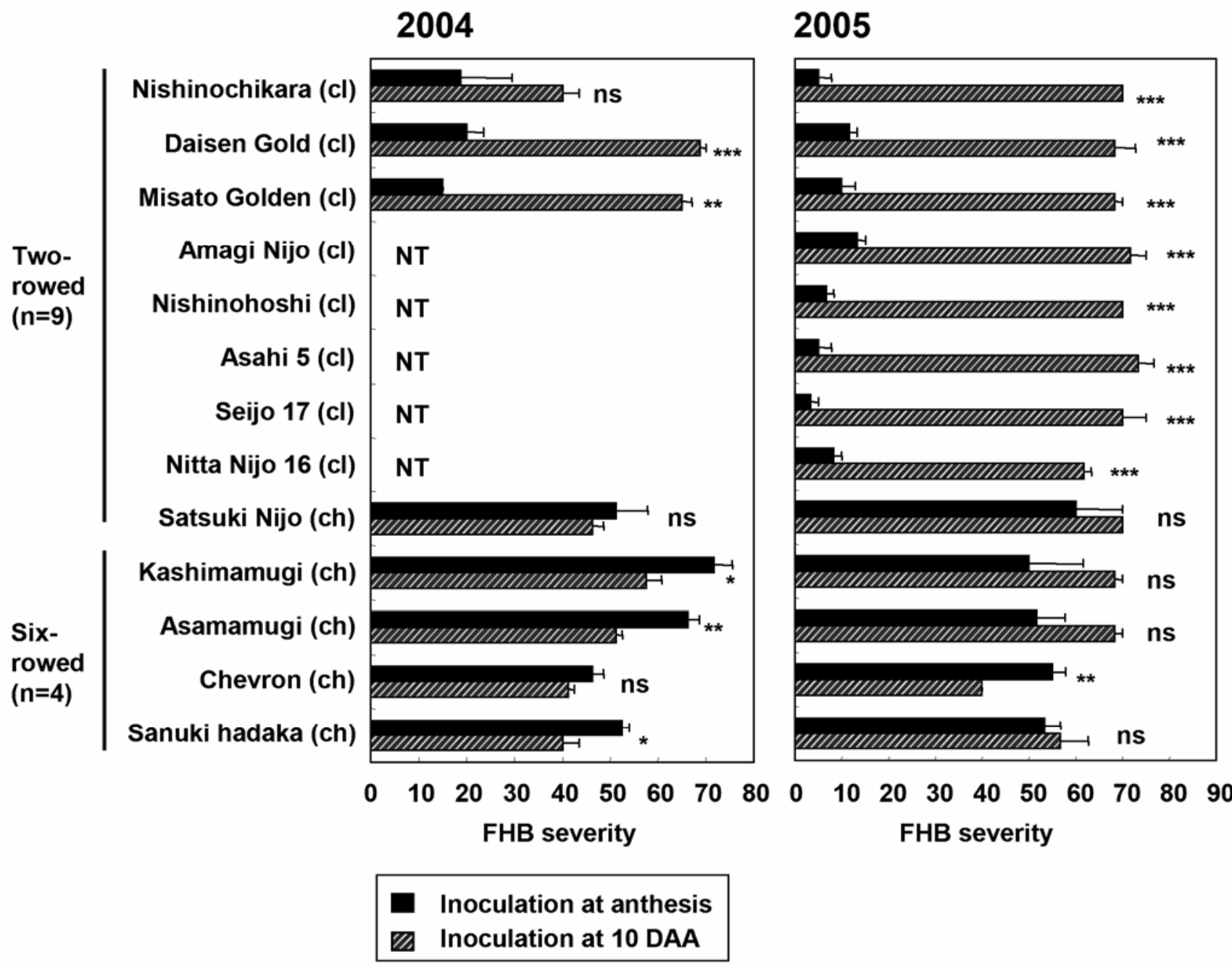

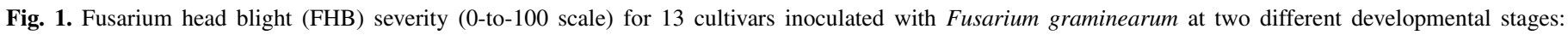

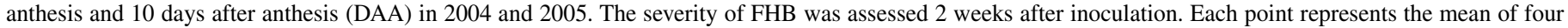

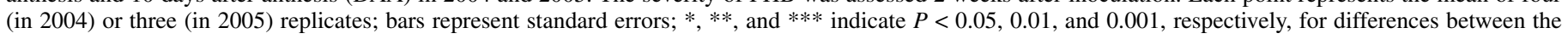
two inoculation times $(t$ test $)$; ns $=$ the difference was not significant; NT = not tested; $\mathrm{cl}=$ cleistogamous cultivar; and ch $=$ chasmogamous cultivar. 
2004

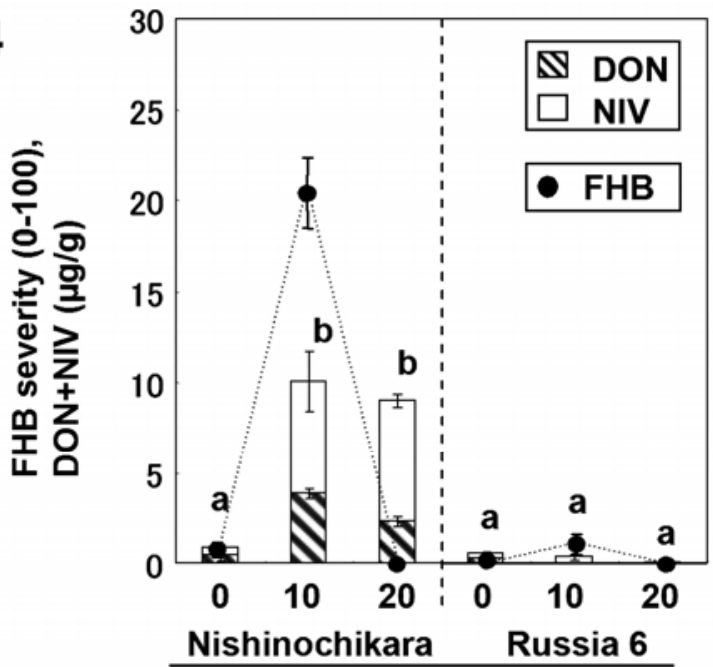

(Two-rowed, cleistogamous)

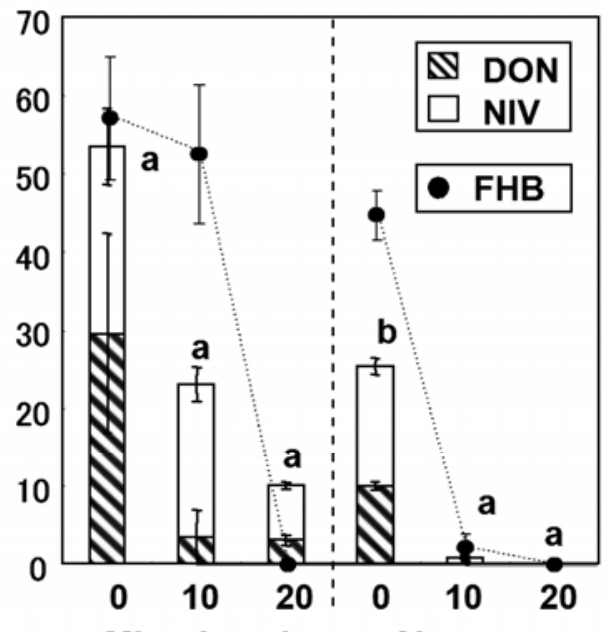

Minorimugi Chevron

(Six-rowed, chasmogamous)

\section{5}

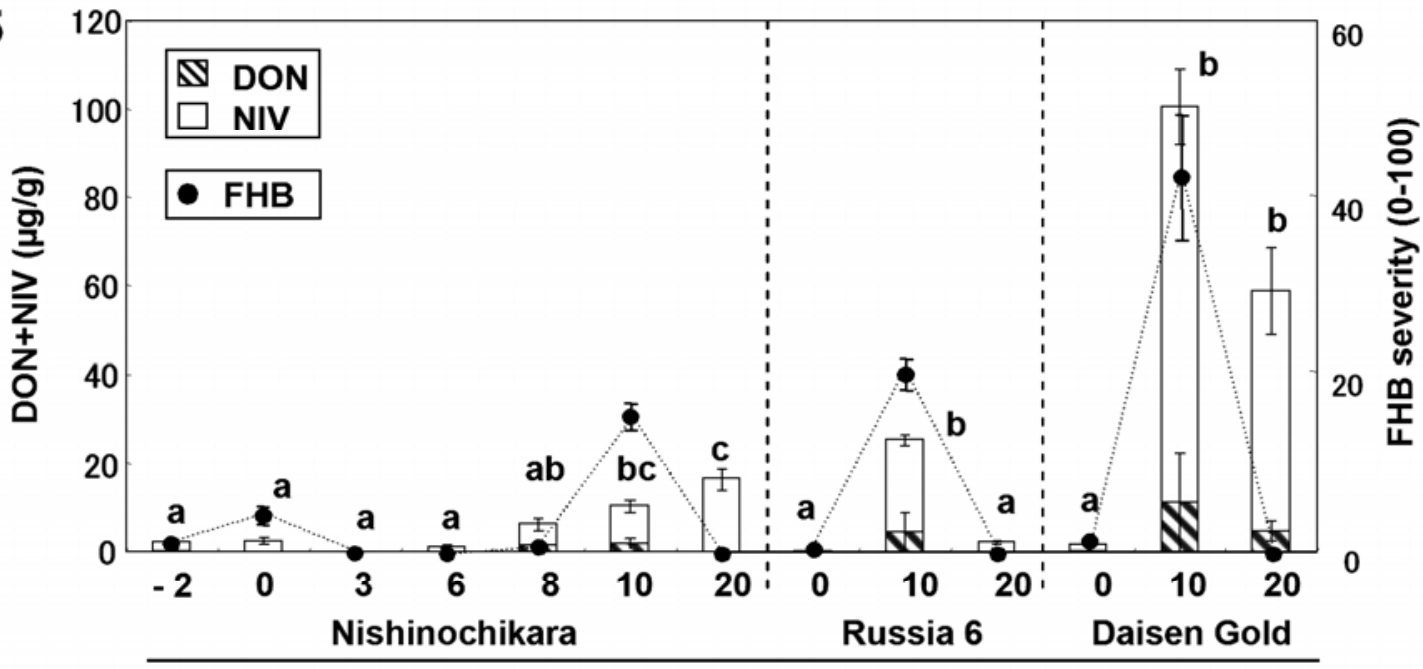

(Two-rowed, cleistogamous)

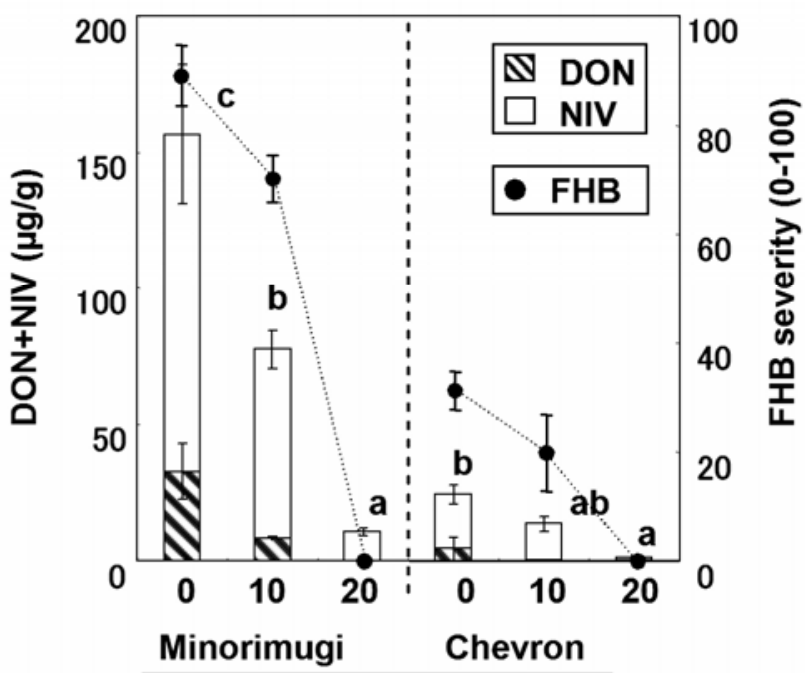

(Six-rowed, chasmogamous)

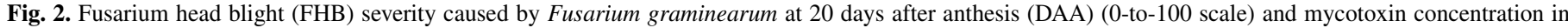

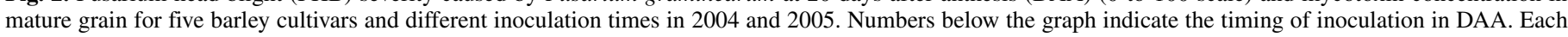

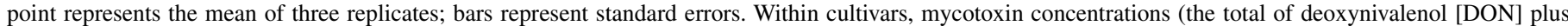

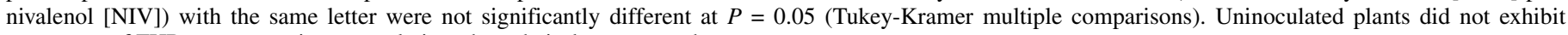
symptoms of FHB or mycotoxin accumulation; thus, their data are not shown. 
2004) or three (in 2005) pots (replicates) for each timing of inoculation and each cultivar were tested in a completely randomized design. The spikes were spray inoculated with the inoculum at $\approx 20 \mathrm{ml} /$ pot at the respective developmental stages between 1500 and $1800 \mathrm{~h}$ each day, and then were incubated overnight in a greenhouse at 18 to $25^{\circ} \mathrm{C}$ and 95 to $100 \%$ humidity provided by humidifiers. After inoculation, the plants were placed in a greenhouse $\left(18\right.$ to $\left.25^{\circ} \mathrm{C}\right)$ equipped with a sprinkler system that produced a fine mist intermittently to keep the spikes moist during the test. The severity of FHB was assessed 2 weeks after inoculation on a scale of 0 to $100(0,2,5,10,20,30,40,50,60,70,80$, 90 , and 100) according to visual rating of the percentage of infected florets. The evaluation was based on the average severity of typical diseased spikes in the pots; late or small spikes were ignored.

Effect of timing of infection on mycotoxin accumulation and FHB severity in barley cultivars (experiment 2). The experiment was conducted in Koshi $\left(32.5^{\circ} \mathrm{N}, 130.4^{\circ} \mathrm{E}\right)$, Kumamoto, Japan, in 2004 and 2005. Five barley cultivars differing in row type and flowering type (Fig. 2) were used to examine the effect of the timing of $F$. graminearum infection on mycotoxin accumulation and the severity of FHB. 'Russia 6' was previously reported as highly resistant to FHB (36), and 'Minorimugi' is one of the leading barley cultivars in Japan. The other three cultivars also were tested in experiment 1.

The cultivars were seeded in $18-\mathrm{cm}$-diameter plastic pots (six plants per pot) at the beginning of December 2003 and 2004, and grown in a greenhouse under natural light until inoculation (April to May 2004 and 2005). The potting soil was prepared by mixing 30 liters of volcanic ash soil, 25 liters of vermiculite, 5 liters of compost, 4 liters of leaf mold, and $50 \mathrm{ml}$ of calcium magnesium carbonate. Each pot contained $\approx 2$ liters of mixed soil and was fertilized with $2.5 \mathrm{~g}$ of $\mathrm{N}-\mathrm{P}_{2} \mathrm{O}_{5}-\mathrm{K}_{2} \mathrm{O}$ at $8-8-8,2 \mathrm{~g}$ of fused magnesium phosphate before seeding, and $1.4 \mathrm{~g}$ of $\mathrm{N}-\mathrm{P}_{2} \mathrm{O}_{5}-\mathrm{K}_{2} \mathrm{O}$ at 8-8-8 at the stem elongation stage (ZGS 30 to 31 ). Late tillers of each plant were removed at the heading stage, leaving 7 to 12 similar-stage spikes per pot.

A mixture of two isolates of $F$. graminearum differing in trichothecene chemotype (i.e., H-3 of the DON chemotype and NIV-2 of the NIV chemotype) was used as the inoculum for experiment 2. Macroconidia of these isolates were produced as in experiment 1 . The macroconidia concentration was provisionally adjusted to $2 \times 10^{6}$ spores $/ \mathrm{ml}$ and then stored at $-20^{\circ} \mathrm{C}$ until use. On the days of inoculation, the macroconidia suspensions of the two isolates were thawed and diluted to $5 \times 10^{5}$ spores $/ \mathrm{ml}$ and then mixed in equal volumes. Finally, Tween 20 was added to a final concentration of $0.2 \mathrm{ml} /$ liter.

Inoculation was performed at three developmental stages: anthesis (0 DAA), 10 DAA, and 20 DAA, with the exception of cv. Nishinochikara in 2005, which was inoculated at seven developmental stages: 2 days before anthesis and 3, 6, and 8 DAA, in addition to the above three stages. For each pot, 0 DAA was defined as the day on which all spikes in the pot reached anthesis. Because late tillers were removed at the heading stage, the spikes in each pot flowered almost uniformly. The developmental stages at 10 and 20 DAA corresponded to the early-milk (ZGS 73) and late-milk (ZGS 77) stages, respectively. Three pots (replicates) for each timing of inoculation for each cultivar were tested in a completely randomized design. For each cultivar, a control consisting of three pots of uninoculated plants also was prepared.

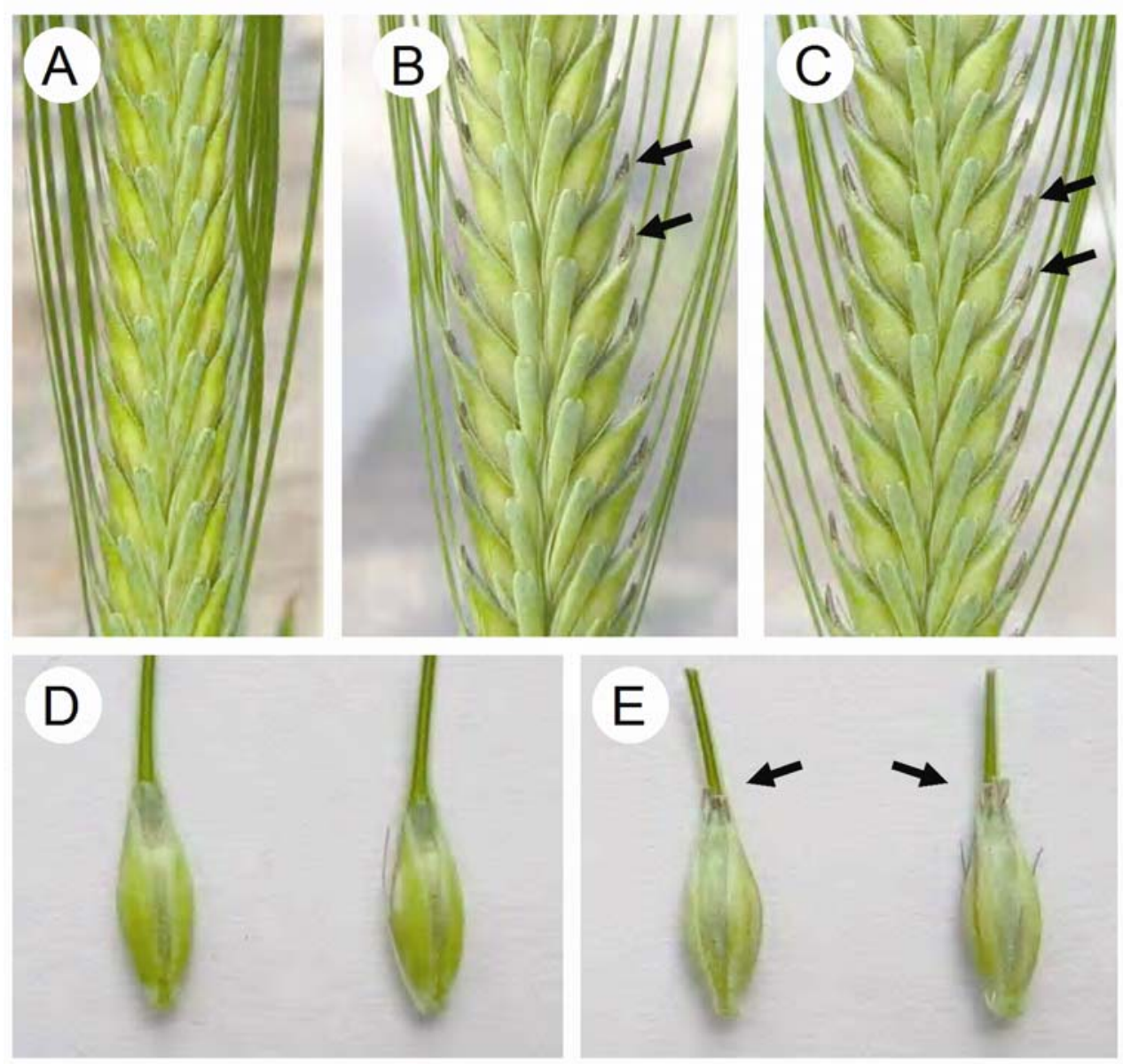

Fig. 3. Extrusion of the spent anthers in the cleistogamous barley cv. Nishinochikara. Spikes at A, 2 days after anthesis (DAA); B, 8 DAA; and C, 10 DAA. Appearance of the ventral side of florets at D, 6 DAA and E, 9 DAA. Anthers were still contained within the closed florets at 6 DAA; most anthers were partially extruded at 8 DAA; most anthers were completely extruded at 10 DAA. Arrows indicate the extruded spent anthers. 
Inoculation was performed between 1400 and $1600 \mathrm{~h}$ on each designated day. Spikes of the potted plants were spray inoculated with the inoculum at $20 \mathrm{ml} /$ pot using an airbrush (Piece Com PCWIDE 308; Olympos, Nabari, Mie, Japan). Thereafter, the inoculated plants were maintained for 6 days under natural light in a greenhouse equipped with a sprinkler system that intermittently produced a fine mist to keep the inoculated spikes moist. The plants then were returned to the original greenhouse in which they were grown. The mean temperatures in the second and original greenhouses during the test period were 18.4 and $20.6^{\circ} \mathrm{C}$ in 2004 and 19.7 and $21.9^{\circ} \mathrm{C}$ in 2005 , respectively. At 20 DAA, the severity of FHB was assessed. All spikes in each pot were evaluated individually on a scale of 0 to 100 , as in experiment 1 ., and pot averages were calculated.

The spikes in each pot were harvested at maturity (32 to $35 \mathrm{DAA}$ ) and dried at $70^{\circ} \mathrm{C}$ for $24 \mathrm{~h}$. After drying, the spikes were threshed carefully so that no small or light kernels were lost. The percentage of discolored kernels (DK), which showed tan to dark brown discoloration, was determined for each grain sample. DK were classified into two groups according to the proportion of discolored area, where DK1 $=>50 \%$ of the grain surface was discolored and DK2 $=10$ to $50 \%$ of the grain surface was discolored. After the evaluation of DK, the DON and NIV contents of each grain sample were analyzed. The grain samples were analyzed for mycotoxins using the previously described enzymelinked immunosorbent assay system (45) by the KM Assay Center, Kyowa Medex Company Limited (Nagaizumi-cho, Shizuoka, Japan).

Statistical analyses. Statistical analyses were performed using Statview (version J5.0; SAS Institute, Cary, NC). For experiment 1 , evaluation of the differences in FHB resistance at the two developmental stages for each cultivar, differences in the mean severity of FHB were tested using a $t$ test for each year. For experiment 2, evaluation of the effect of the timing of infection on the mycotoxin levels in the mature grain, we compared levels within cultivars using Tukey-Kramer multiple comparisons for each year.

\section{RESULTS}

Evaluation of FHB resistance at two developmental stages in barley cultivars (experiment 1). In the cleistogamous cultivars, anthers did not protrude at anthesis; however, as the grain developed after pollination, the spent anthers were extruded from the tip of the floret between the tip of the palea and lemma (Fig. 3 ). Spent anther extrusion began at $\approx 7$ DAA and was completed by 10 DAA.

When inoculated at anthesis, the tested cultivars showed a wide range in the severity of FHB in both years (Fig. 1). The level of disease severity caused by inoculation at anthesis differed depending on the flowering type: cleistogamous cultivars showed good FHB resistance, with severity of 15.0 to 20.0 in 2004 and 3.3 to 13.3 in 2005 , whereas chasmogamous cultivars showed relatively high severity of FHB, regardless of row type, with FHB scores of 46.3 to 71.7 in 2004 and 50.0 to 60.0 in 2005 . When inoculated at 10 DAA, however, the cultivars' responses differed from those obtained by inoculation at anthesis: cleistogamous cultivars were severely diseased, with severity of 40.0 to 68.8 in 2004 and 61.7 to 73.3 in 2005, as were chasmogamous cultivars, with FHB scores of 40.0 to 57.5 in 2004 and 40.0 to 70.0 in 2005.

A difference in resistance between the two developmental stages occurred in cleistogamous cultivars in both years. The severity of FHB following inoculation at 10 DAA was significantly higher than with inoculation at anthesis for each cultivar, with the exception of Nishinochikara in $2004(P=0.11)$. In cultivars inoculated at 10 DAA, symptoms of FHB, a brown discoloration, usually appeared around the tips of florets, where the spent anthers were extruded, after which the discoloration progressed toward the bases of the florets. In contrast, in chasmogamous cultivars, the severity of FHB did not differ between inoculations done at 0 and 10 DAA, or the severity was higher in the former, although no differences were observed consistently in both years.

Effect of the timing of infection on mycotoxin accumulation and FHB severity in barley cultivars (experiment 2). In this experiment, the severity of FHB was assessed at 20 DAA for all plants, which is the commonly used timing for FHB evaluation in Japan; therefore, the severity of FHB in plants inoculated at 20 DAA was zero for all five cultivars. Following 20 DAA, the spike color gradually faded and turned brownish due to natural senescence, irrespective of cultivar, masking the appearance of the disease symptoms. As in experiment 1 , anther extrusion in cleistogamous cultivars began at $\approx 7$ DAA and was completed by 10 DAA.

The effect of the timing of infection on both mycotoxin accumulation and the severity of FHB differed between the tworowed cleistogamous cultivars and the six-rowed chasmogamous cultivars (Fig. 2). Chasmogamous cultivars exhibited the highest severity of FHB when inoculated at 0 DAA. In contrast, cleistogamous cultivars were only slightly diseased when inoculated at 0 DAA and exhibited the highest severity of FHB when inoculated at 10 DAA, with the exception of Russia 6 in 2004, which had few symptoms when inoculated at 10 DAA. Thus, the timing of infection affected the severity of FHB differentially among cultivars that differed in flowering type. This is consistent with the results of experiment 1 .

The total concentration of DON and NIV was highest with inoculation at 0 DAA for chasmogamous cultivars, similar to the severity of FHB. In contrast, the mycotoxin concentration in cleistogamous cultivars was significantly higher with inoculation at 10 DAA compared with 0 DAA, with the exception of Russia 6 in 2004, in which little mycotoxin accumulated when inoculated at 10 DAA (Fig. 2).

Data for Nishinochikara in 2005 indicated that the change in this cultivar's response to inoculation between 0 and 10 DAA actually occurred between 6 and 10 DAA because the severity of FHB and mycotoxin concentration with inoculation at 10 DAA were significantly higher than with inoculation at 6 DAA and earlier. At 6 DAA, all anthers were still inside the closed florets; by 8 DAA, most anthers had been partially extruded; at 10 DAA, most anthers had been completely extruded (Fig. 3).

Inoculation at 20 DAA did not cause clear symptoms of FHB in any cultivar. However, it did result in accumulation of DON and NIV. The cleistogamous cultivars, in particular, except for Russia 6 , accumulated high levels of DON and NIV with inoculation at 20 DAA (DON + NIV: 9.0 to $58.8 \mu \mathrm{g} / \mathrm{g}$ ), and the total concentration of DON and NIV in these cultivars was significantly higher than when inoculated at 0 DAA. The chasmogamous cv. Minorimugi also accumulated high levels of DON and NIV after inoculation at 20 DAA (DON + NIV: 10.1 and $10.7 \mu \mathrm{g} / \mathrm{g}$ in 2004 and 2005, respectively); however, this concentration was lower than that with inoculation at 0 and $10 \mathrm{DAA}$, although the difference did not reach significance in 2004 ( $P=0.059$ by analysis of variance). For chasmogamous cultivars, both the severity of FHB and mycotoxin concentrations declined when infection timing was delayed.

The change in the percentage of DK with the later timing of infection (Fig. 4) was associated with the change in the severity of FHB (Fig. 2), except in cvs. Nishinochikara and Daisen Gold in 2005. These two cultivars did not show distinct symptoms of FHB on the spikes following inoculation at 20 DAA in either year (Fig. 2 ), although the harvested grain samples in 2005 contained 34.5 and $49.6 \%$ DK, respectively; however, most of these were stained only around the base of the kernel and were graded as DK2 (10 to $50 \%$ of the grain surface discolored) (Fig. 4). For all cultivars, the change in the percentage of DK in 2005 was similar to the pattern of mycotoxin accumulation, rather than the severity of FHB (Figs. 2 and 4). 
Unlike in 2005, the change in the percentage of DK in 2004 was associated with the change in the severity of FHB. The kernels from plants inoculated at 20 DAA accumulated high levels of mycotoxin without clear disease symptoms being present (Figs. 2 and 4). The cvs. Nishinochikara and Minorimugi accumulated mycotoxin (mean DON + NIV) at 9.0 and $10.1 \mu \mathrm{g} / \mathrm{g}$, respectively, following inoculation at 20 DAA in 2004, without displaying clear disease symptoms on the spike or the kernels (Figs. 2, 4, and 5).

\section{DISCUSSION}

To our knowledge, this is the first investigation of the effect of the timing of Fusarium infection on mycotoxin accumulation and severity of FHB in barley. We investigated the accumulation of both DON and NIV because they can often co-occur $(25,37$, $38,43,44)$.

The timing of inoculation had different effects on the severity of FHB between the two barley flowering types. Cleistogamous cultivars exhibited good resistance when inoculated at anthesis, but susceptibility increased drastically with inoculation at 10 DAA. In contrast, chasmogamous cultivars were susceptible to inoculation at anthesis, and this susceptibility was retained or de- clined at 10 DAA. These results were similar in two separate experiments. Furthermore, in experiment 2, the mycotoxin concentration in harvested grain was affected by the timing of infection in a manner similar to the severity of FHB with inoculations between 0 and 10 DAA; the mycotoxin concentration in the tworowed cleistogamous cultivars was much higher with inoculation at 10 than at 0 DAA in most cases. Although we tested only five cultivars for mycotoxin accumulation, this increase in accumulation of mycotoxins associated with susceptibility to FHB after anthesis likely is common in two-rowed cleistogamous cultivars, because an increase in susceptibility to FHB occurred in all tworowed cleistogamous cultivars tested in experiment 1 .

The drastic increase in susceptibility to FHB and mycotoxin accumulation in the two-rowed cleistogamous cultivars appears attributable to the extrusion of the spent anthers, which occurs after anthesis, because (i) previous studies with wheat have shown that initial Fusarium infection occurs on fully or partially extruded anthers (26) and that anther extracts enhance fungal growth $(5,33)$, suggesting that anthers may play a fundamental role in infection by $F$. graminearum and other Fusarium spp. (26,33); (ii) recent studies of near-isogenic barley lines indicate that cleistogamy contributes to resistance to FHB at anthesis (42), suggesting
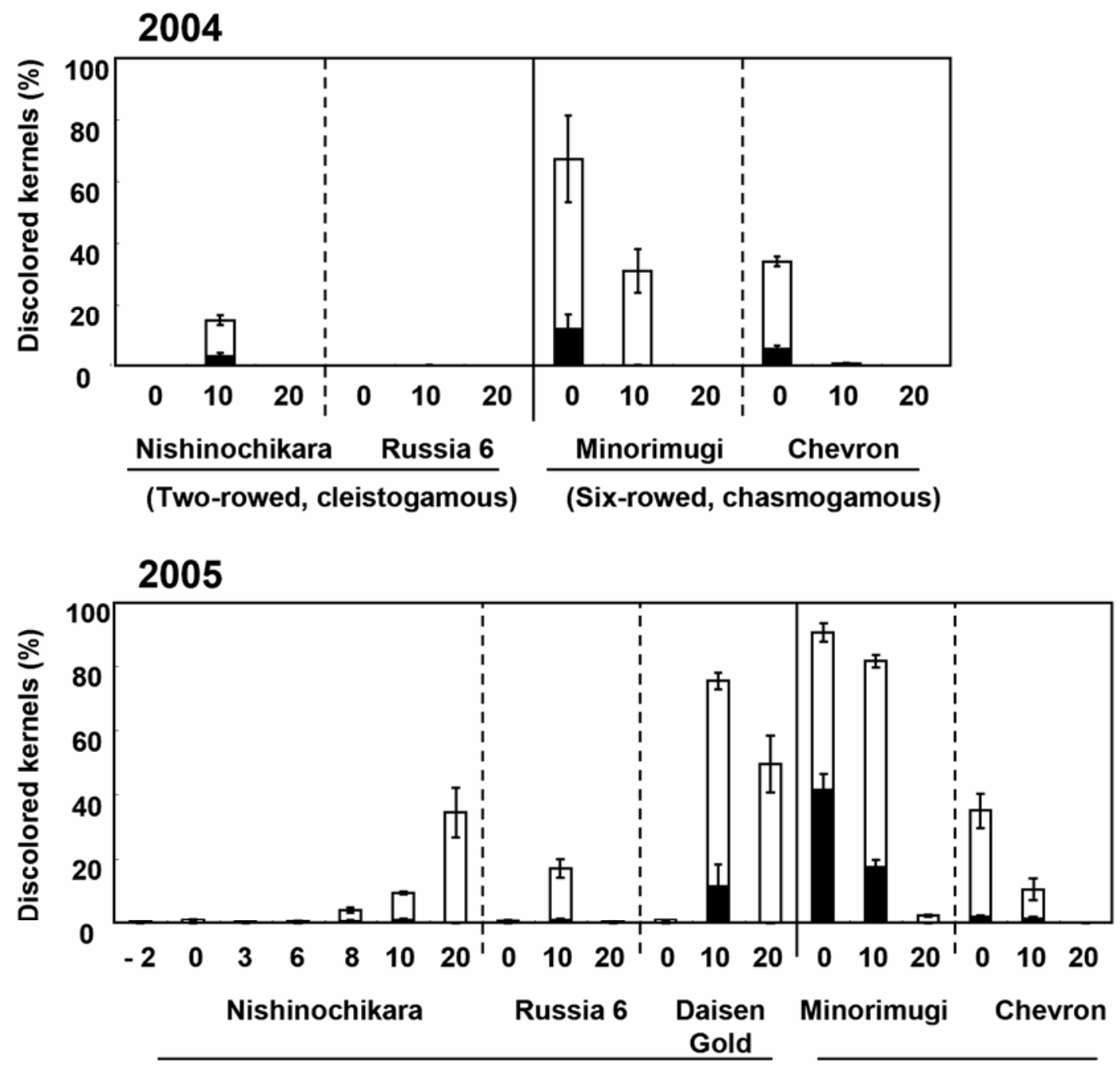

(Two-rowed, cleistogamous)

(Six-rowed, chasmogamous)

DK1: more than $\mathbf{5 0} \%$ of the grain surface discolored DK2: $\mathbf{1 0 - 5 0} \%$ of the grain surface discolored

Fig. 4. Percentage of discolored kernels (DK) for five barley cultivars and different inoculation times with Fusarium graminearum in 2004 and 2005 . Numbers below the graph indicate the timing of inoculation in days after anthesis (DAA). Each point represents the mean of three replicates; bars represent standard errors. 
that the extrusion of the anthers in barley also may promote infection by $F$. graminearum; (iii) in cleistogamous cultivars inoculated at 10 DAA, symptoms of FHB usually appeared around the tips of the florets, where the spent anthers extruded and usually remained; and (iv) the increase in susceptibility to FHB in cv. Nishinochikara in 2005 occurred between 6 and 10 DAA, during which time the extrusion of the anthers actually occurred. It is likely that the extruded spent anthers provide an initial base for the colonization of $F$. graminearum in resistant cleistogamous cultivars.

Lewandowski et al. (14) recently reported that anthers may be less important for infection by $F$. graminearum in barley than in wheat, based on their field inoculation experiments using the susceptible six-rowed cv. Robust. In their study, florets with colonized anthers were not observed frequently following inoculation, although some anthers were colonized and provided a bridge for fungal entry into florets, as previously observed in wheat (26). It is possible that the importance of the anthers in infection by $F$. graminearum differs with barley cultivars that differ in resistance or flowering type. Further studies are needed to elucidate the infection pathway at different developmental stages in resistant cleistogamous cultivars, as well as in fully susceptible cultivars.

Barley usually undergoes anthesis after heading (2). However, in some regions where barley is grown, anthesis occurs while the spike is still enclosed within the flag leaf sheath $(16,32)$. The experiments by Lewandowski et al. (14) cited above were performed in the Upper Midwest region of the United States (14), where barley plants commonly flower before heading (32). It is not described when flowering actually occurred in their study; however, if it occurred before heading, this also may have caused the apparent inconsistence between their and our studies. In the situations where anthesis occurs before heading, the importance of the anthers in infection by $F$. graminearum and the possible contribution of flowering type and anther extrusion to changes in resistance to FHB after anthesis might differ from that described in the present study. Nevertheless, the resistance to FHB may be greatly affected by the timing of anther extrusion when this occurs after heading.

The accumulation of DON and NIV in the grain resulted from infection events up to 20 DAA, even when such late infection did not display distinct symptoms of FHB. Thus, even if the severity of FHB assessed in the field appears absent or very low, DON and NIV can accumulate in the mature grain as a result of late infections. In addition, the mycotoxin levels found are not necessarily low. This risk appears to be barley cultivar dependent, but is not related to the flowering type or row type. Among the tested cultivars, Nishinochikara, Daisen Gold, and Minorimugi appear to be at high risk of mycotoxin contamination following late infection, whereas the cvs. Russia 6 and Chevron appear to be at low risk. For such late infection, infection courts other than extruded anther may play an important role. There may exist resistance mechanisms for such late infection which are unrelated to spike morphology and independent of resistance at early stages. Russia 6 and Chevron may possess such "late resistance." Remarkably, the Japanese two-rowed cleistogamous cvs. Nishinochikara and Daisen Gold accumulated comparable levels of mycotoxins with inoculation at 10 and $20 \mathrm{DAA}$, and these levels were much higher than those accumulated following inoculation at anthesis. Thus, late infection without visible symptoms of FHB cannot be ignored in terms of there being a risk of mycotoxin contamination.

Furthermore, high levels of DON and NIV contamination in barley grain resulting from late infection may even lack visible symptoms on the grain. The cvs. Nishinochikara and Minorimugi, which accumulated high levels of mycotoxin when inoculated at 20 DAA, exhibited some discoloration of the grain in 2005 but not in 2004. It is not clear what caused this difference between years; however, minor differences in environmental conditions or other microorganisms that also cause kernel discoloration in barley (20) may have been involved.

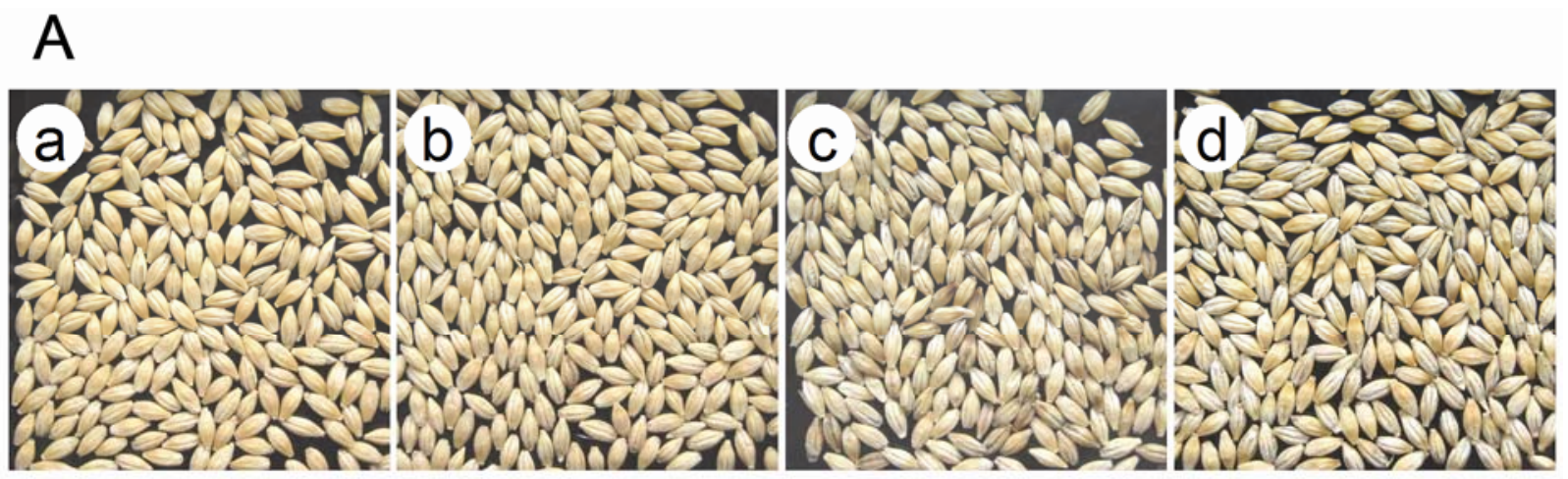

B
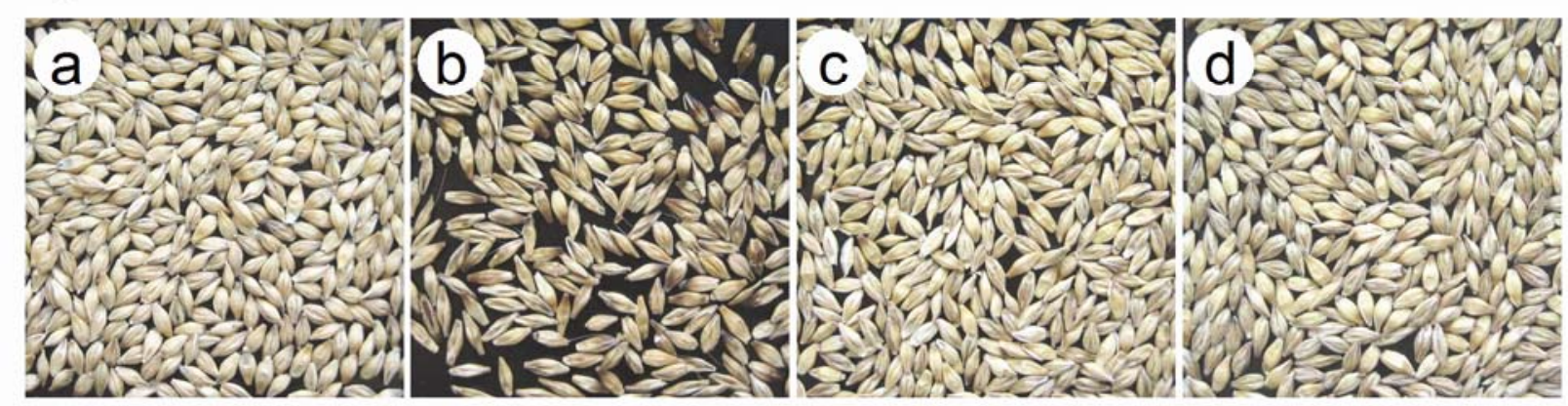

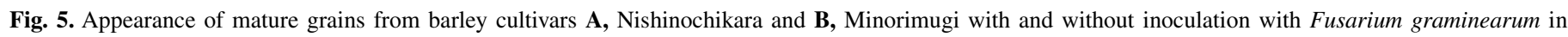

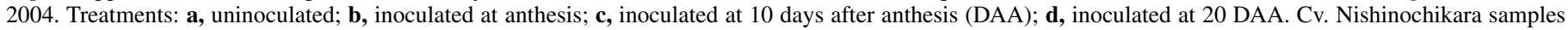

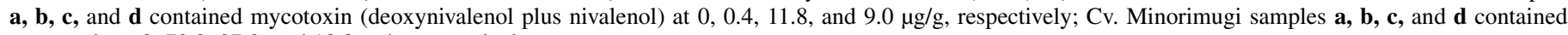
mycotoxin at $0,70.9,27.0$, and $10.3 \mu \mathrm{g} / \mathrm{g}$, respectively. 
The relationship between mycotoxin concentrations and visual symptoms of FHB on spikes and grain is weak or inconsistent in both barley $(3,29,39)$ and wheat $(15,19,23,24,31)$. For barley, this can be attributed in part to the presence of other pathogens that cause symptoms similar to those of FHB $(3,29)$. In addition, the present study demonstrated that differences in the timing of infection also can contribute to inconsistencies in the relationship between mycotoxin concentration levels and disease severity, in both the spike and grain. Barley kernels that are normal in appearance may contain high levels of DON $(32,39)$. Our data suggest that such cases may be caused by late infection.

There currently are no robust control measures to manage either FHB or mycotoxin contamination in barley and wheat. Fungicide application is one measure available to reduce the risk; however, this has not been highly effective or consistent in barley or wheat $(8,11,18,32)$. Although studies of chemical control of FHB in barley are limited, a fungicide generally is applied at or near anthesis $(6,10,11)$, similar to its application in wheat. In Japan, fungicide application for control of FHB in barley usually is performed around the time of anthesis, which occurs a few days after the full heading stage. Thus, the time of anthesis is regarded as the most important stage for Fusarium infection in barley, probably following the example of wheat. However, we have demonstrated that the most critical time for $F$. graminearum infection and subsequent mycotoxin accumulation in barley differs among cultivars and appears to be associated with the flowering type. For two-rowed cleistogamous cultivars in Japan, infection that occurs after the extrusion of spent anthers would be much more important than infection around anthesis. Our findings suggest that the optimal timing for chemical control of FHB and mycotoxin contamination in barley will depend on the cultivar. For cleistogamous cultivars, the efficacy of fungicide applications is expected to be improved by changing the timing of application to near the extrusion of spent anthers. Fungicide trials to test this prediction are currently underway.

\section{ACKNOWLEDGMENTS}

This study was conducted as part of the Integrated Research Program for Providing Fresh and Delicious "Brand Nippon" Agricultural Products, and as part of the Integrated Research Program for Functionality and Safety of Food Toward an Establishment of Healthy Diet. Funding was provided by the Ministry of Agriculture, Forestry, and Fisheries of Japan. We thank the staff of the research team for FHB control at KONARC for technical assistance.

\section{LITERATURE CITED}

1. Atanasoff, D. 1920. Fusarium-blight (scab) of wheat and other cereals. J. Agric. Res. 20:1-40.

2. Briggs, D. 1978. Barley. Chapman and Hall, London.

3. Choo, T. M., Martin, R. A., Ho, K. M., Shen, Q., Fedak, G., Savard, M., Voldeng, H., Falk, D. E., Etienne, M., and Sparry, E. 2004. Fusarium head blight and deoxynivalenol accumulation of barley in eastern Canada: Cultivar response and correlation analysis. Plant Dis. 88:837-844.

4. Codex. 2002. Discussion paper on deoxynivalenol. (Agenda Item 16(g), CX/FAC 02/29.) Codex Committee on Food Additives and Contaminants, 34th Session, Rotterdam. Codex Alimentarius Commission FAO/WHO, Rome.

5. Engle, J. S., Lipps, P. E., Graham, T. L., and Boehm, M. J. 2004. Effects of choline, betaine, and wheat floral extracts on growth of Fusarium graminearum. Plant Dis. 88:175-180.

6. Havlova, P., Lancova, K., Vanova, M., Havel, J., and Hajslova, J. 2006. The effect of fungicidal treatment on selected quality parameters of barley and malt. J. Agric. Food Chem. 54:1353-1360.

7. Heta, H., and Hiura, U. 1963. Varietal differences in resistance to head blight, Gibberella zeae (Schw.) Petch. Studies on the disease-resistance in barley. XIII. Nogaku Kenkyu 49:177-187. (In Japanese)

8. Horsley, R. D., Pederson, J. D., Schwarz, P. B., McKay, K., Hochhalter, M. R., and McMullen, M. P. 2006. Integrated use of tebuconazole and Fusarium head blight-resistant barley genotypes. Agron. J. 98:194-197.

9. Ichinoe, M., Kurata, H., Sugiura, Y., and Ueno, Y. 1983. Chemotaxonomy of Gibberella zeae with special reference to production of trichothecenes and zearalenone. Appl. Environ. Microbiol. 46:1364-1369.

10. Ioos, R., Belhadj, A., Menez, M., and Faure, A. 2005. The effects of fungicides on Fusarium spp. and Microdochium nivale and their associated trichothecene mycotoxins in French naturally infected cereal grains. Crop Prot. 24:894-902.

11. Jones, R. K. 2000. Assessments of Fusarium head blight of wheat and barley in response to fungicide treatment. Plant Dis. 84:1021-1030.

12. Kim, H. S., Lee, T., Dawlatana, M., Yun, S. H., and Lee, Y. W. 2003. Polymorphism of trichothecene biosynthesis genes in deoxynivalenoland nivalenol-producing Fusarium graminearum isolates. Mycol. Res. 107:190-197.

13. Lee, T., Han, Y. K., Kim, K. H., Yun, S. H., and Lee, Y. W. 2002. Tri 13 and Tri 7 determine deoxynivalenol- and nivalenol-producing chemotypes of Gibberella zeae. Appl. Environ. Microbiol. 68:2148-2154.

14. Lewandowski, S. M., Bushnell, W. R., and Evans, C. K. 2006. Distribution of mycelial colonies and lesions in field-grown barley inoculated with Fusarium graminearum. Phytopathology 96:567-581.

15. Liu, W., Langseth, W., Skinnes, H., Elen, O. N., and Sundheim, L. 1997. Comparison of visual head blight ratings, seed infection levels, and deoxynivalenol production for assessment of resistance in cereals inoculated with Fusarium culmorum. Eur. J. Plant Pathol. 103:589-595.

16. McCallum, B. D., and Tekauz, A. 2002. Influence of inoculation method and growth stage on Fusarium head blight in barley. Can. J. Plant Pathol. 24:77-80.

17. McCallum, B. D., Tekauz, A., and Gilbert, J. 2004. Reaction of a diverse collection of barley lines to Fusarium head blight. Plant Dis. 88:167-174.

18. McMullen, M., Jones, R., and Gallenberg, D. 1997. Scab of wheat and barley: A re-emerging disease of devastating impact. Plant Dis. 81:13401348.

19. Mesterházy, Á. 2002. Role of deoxynivalenol in aggressiveness of Fusarium graminearum and F. culmorum and in resistance to Fusarium head blight. Eur. J. Plant Pathol. 108:675-684.

20. Miles, M. R., Wilcoxson, R. D., Rasmusson, D. C., Wiersma, J., and Warnes, D. 1987. Influence of genotype and environment on kernel discoloration of Midwestern malting barley. Plant Dis. 71:500-504.

21. Mirocha, C. J., Abbas, H. K., Windels, C. E., and Xie, W. 1989. Variation in deoxynivalenol, 15-deoxynivalenol, 3-acetyldeoxynivalenol and zearalenone production by Fusarium graminearum isolates. Appl. Environ. Microbiol. 55:1315-1316.

22. Parry, D. W., Jenkinson, P., and McLeod, L. 1995. Fusarium ear blight (scab) in small-grain cereals: A review. Plant Pathol. 44:207-238.

23. Paul, P. A., Lipps, P. E., and Madden, L. V. 2005. Relationship between visual estimates of Fusarium head blight intensity and deoxynivalenol accumulation in harvested wheat grain: A meta-analysis. Phytopathology 95:1225-1236

24. Paul, P. A., Lipps, P. E., and Madden, L. V. 2006. Meta-analysis of regression coefficients for the relationship between Fusarium head blight and deoxynivalenol content of wheat. Phytopathology 96:951-961.

25. Placinta, C. M., D’Mello, J. B. F., and Macdonald, A. M. C. 1999. A review of world contamination of cereal grains and animal feeds with Fusarium mycotoxins. Anim. Feed Sci. Technol. 78:21-37.

26. Pugh, G. W., Johann, H., and Dickson, J. G. 1933. Factors affecting infection of wheat heads by Gibberella saubinetii. J. Agric. Res. 46:771797.

27. Rudd, J. C., Horsley, R. D., McKendry, A. L., and Elias, E. M. 2001. Host plant resistance genes for Fusarium head blight: Sources, mechanisms, and utility in conventional breeding systems. Crop Sci. 41:620-627.

28. Ryu, J., Ohtsubo, K., Izumiyama, N., Nakamura, K., Tanaka, T., Yamamura, H., and Ueno, Y. 1988. The acute and chronic toxicities of nivalenol in mice. Fundam. Appl. Toxicol. 11:38-47.

29. Salas, B., Steffenson, B. J., Casper, H. H., Tacke, B., Prom, L. K., Fetch, T. G., Jr., and Schwarz, P. B. 1999. Fusarium species pathogenic to barley and their associated mycotoxins. Plant Dis. 83:667-674.

30. Seo, J. A., Kim, J. C., Lee, D. H., and Lee, Y. W. 1996. Variation in 8keto-trichothecenes and zearalenone production by Fusarium graminearum isolates from corn and barley in Korea. Mycopathologia 134:31-37.

31. Snijders, C. H. A., and Perkowski, J. 1990. Effects of head blight caused by Fusarium culmorum on toxin content and weight of wheat kernels. Phytopathology 80:566-570.

32. Steffenson, B. J. 2003. Fusarium head blight of barley: Impact, epidemics, management, and strategies for identifying and utilizing genetic resistance. Pages 241-295 in: Fusarium Head Blight of Wheat and Barley. K. J. Leonard and W. R. Bushnell, eds. The American Phytopathological Society, St. Paul, MN.

33. Strange, R. N., and Smith, H. 1971. A fungal growth stimulant in anthers which predisposes wheat to attack by Fusarium graminearum. Physiol. Plant Pathol. 1:141-150.

34. Sugiura, Y., Watanabe, Y., Tanaka, T., Yamamoto, S., and Ueno, Y. 1990. Occurrence of Gibberella zeae strains that produce both nivalenol and 
deoxynivalenol. Appl. Environ. Microbiol. 56:3047-3051.

35. Sutton, J. C. 1982. Epidemiology of wheat head blight and maize ear rot caused by Fusarium graminearum. Can. J. Plant Pathol. 4:195-209.

36. Takeda, K., and Heta, H. 1989. Establishing the testing method and a search for the resistant varieties to Fusarium head blight in barley. Jpn. J. Breed. 39:203-216. (In Japanese with English summary)

37. Tanaka, T., Hasegawa, A., Yamamoto, S., Lee, U. S., Sugiura, Y., and Ueno, Y. 1988. Worldwide contamination of cereals by the Fusarium mycotoxins nivalenol, deoxynivalenol, and zearalenone. 1. Survey of 19 countries. J. Agric. Food Chem. 36:979-983.

38. Tanaka, T., and Sugiura, Y. 2003. Levels and pattern of contamination with trichothecenes in cereal grains and their risk assessment. Mycotoxins 53:119-121.

39. Tekauz, A., McCallum, B., and Gilbert, J. 2000. Review: Fusarium head blight of barley in western Canada. Can. J. Plant Pathol. 22:9-16.

40. Ueno, Y., Aikawa, Y., Okumura, H., Sugiura, Y., Nakamura, K., Masuma, R., Tanaka, T., Young, C. J., and Savard, M. E. 1997. Trichothecenes produced by Fusarium species Fn 2B. Mycotoxins 45:25-31.

41. Yoshida, M., Kawada, N., and Tohnooka, T. 2003. The "pot-plant" method provides accurate, stable testing of the resistance to Fusarium head blight in barley. Bull. Natl. Inst. Crop Sci. 3:1-19. (In Japanese with English abstract)

42. Yoshida, M., Kawada, N., and Tohnooka, T. 2005. Effect of row type, flowering type and several other spike characters on resistance to Fusarium head blight in barley. Euphytica 141:217-227.

43. Yoshizawa, T. 1997. Geographic difference in trichothecene occurrence in Japanese wheat and barley. Bull. Inst. Compr. Agric. Sci. Kinki Univ. 5:23-30.

44. Yoshizawa, T., and Jin, T. Z. 1998. Trichothecene occurrence in Japanese wheat and barley-its characteristics. Mycotoxins 47:15-18.

45. Yoshizawa, T., Kohno, H., Ikeda, K., Shinoda, T., Yokohama, H., Morita, K., Kusada, O., and Kobayashi, Y. 2004. A practical method for measuring deoxynivalenol, nivalenol, and T-2 + HT-2 toxin in foods by an enzyme-linked immunosorbent assay using monoclonal antibodies. Biosci. Biotechnol. Biochem. 68:2076-2085.

46. Zadoks, J. C., Chang, T. T., and Konzak, C. F. 1974. A decimal code for the growth stages of cereals. Weed Res. 14:415-421.

47. Zhou, X., Chao, M., and Liang, X. 1991. Screening and testing of barley varieties for scab resistance. Acta Phytophylactica Sin. 18:261-265. (In Chinese with English summary). 